\title{
Las dos caras de las elecciones
}

\author{
Ana Mercedes Saiz Valenzuela
}

En primer término se hace un análisis del sistema electoral en el que interactúan de manera simultánea una rama ciudadana y una rama técnica. En una segunda parte se estudia el contexto y los puntos más relevantes de la reforma electoral de 2008. En un tercer apartado se examina el sistema para la fiscalización de los recursos que erogaron los partidos políticos, en específico se enuncian las particularidades de la aplicación de estas normas durante el Proceso Electoral Federal 20I2-20I3. Por último, se hace un análisis detallado de la norma en la que se materializó el reclamo de la izquierda "voto por voto" y la manera en que distintas autoridades electorales la aplicaron en tres casos concretos.

PALABRAS CLAVE: sistema electoral, recuento, cómputo, partido político, voto, ciudadanización, democracia

\section{Two Sides of the Election}

The construction of the electoral system in which the citizen and the technical branches interact simultaneously is analyzed in the first part. In the second part, the context and the most relevant aspects of the electoral reform of 2008, that included prohibiting the sale and purchase of radio and television time, are studied.The third part examines the audit system for the resources spent by the political parties, and the use of these norms within the electoral process 2012-20I3. The last part is a detailed analysis of three cases involving the interpretation and application of the rule that embodied the leftish claim "voto por voto". After studying the three cases and the way the recount rule was interpreted and enforced by the electoral authorities, we could see that it was favorable to political parties and not citizen oriented.

KEYWORDS: electoral system, recount, tally, political party, vote, citizen-centered, democracy 


\section{INTRODUCCIÓN}

A pesar de que el Instituto Federal Electoral (IFE) logró en julio de 2012 la realización de una elección presidencial y aplicó una reforma electoral que le impuso grandes retos, un sector de la población percibe que las autoridades electorales no cumplieron cabalmente con despejar las dudas sobre la compra y la coacción del voto, la influencia de los medios de comunicación sobre los electores y, en general, de la equidad de la contienda. El sector al que me refiero no está formado sólo por simpatizantes de Andrés Manuel López Obrador, candidato que obtuvo el segundo lugar en las dos últimas elecciones presidenciales, 2006 y 2012. Por ello, no puede considerarse como un fenómeno relacionado únicamente con la no aceptación de la derrota electoral.

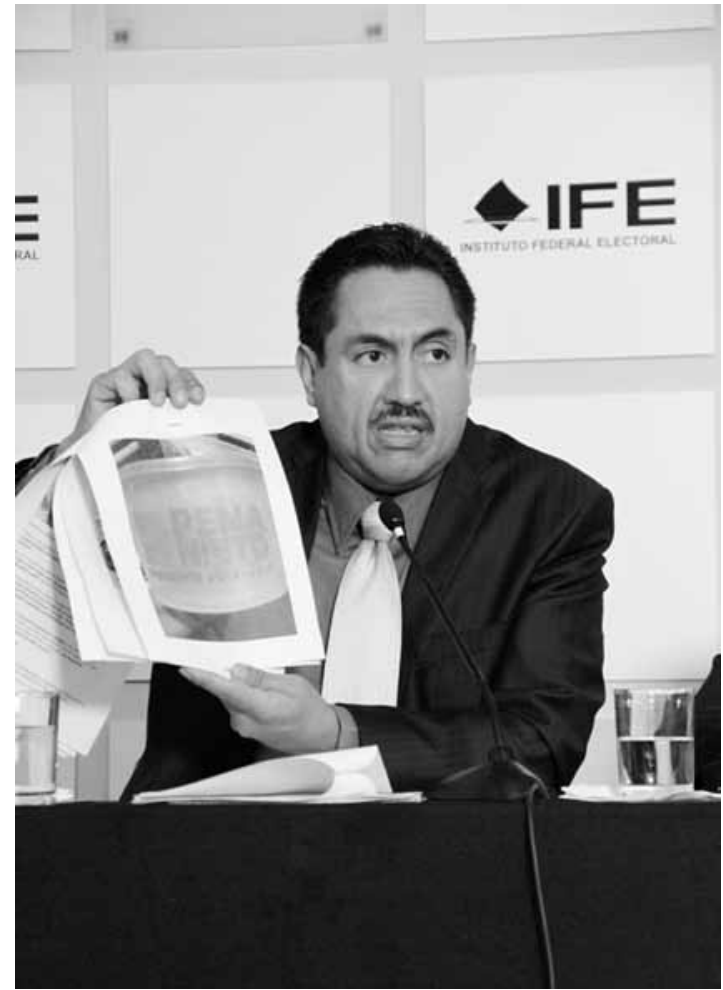

Camerino Márquez muestra los objetos y souvenirs que regalan los priistas a cambio del voto ciudadano, 6 de mayo de 2012 .
Según la encuesta publicada por Consulta Mitofsky en octubre de 2012 sobre la confianza de los mexicanos en las instituciones, el IFE registró una calificación de 6.7, confianza media, junto a otros cuatro organismos de los 15 evaluados. El Instituto genera "mucha confianza" en $19.8 \%$ de las personas, "regular" en un poco más de $50 \%$ y "nada de confianza" entre 20\% de los mexicanos. Ahora bien, Consulta Mitofsky presenta la evolución de la confianza en el IFE a lo largo de cinco años: $24.6 \%$ tenía "mucha confianza" en 2008, en 2009 esta cifra era de 23.4\%, en 2010 fue de $20.6 \%$ y en 2011 de $13.4 \%$. Como se observa, en 2012 hay un repunte, aunque debemos tomar en cuenta la opinión de quienes tienen "poco o nada" de confianza en el IFE, cuyos porcentajes aumentan consistentemente de $17.6 \%$ en 2009 y hasta $21.1 \%$ en 2012 (Consulta Mitofsky, 2012). Aunque los datos citados son muy recientes, no podemos dejar de mencionar que entre 2000 y 2001 las opiniones favorables de la población sobre el IFE llegaron a $77 \%$ (CESOP, 2003).

\section{EL DISEÑO EN EQUILIBRIO DEL SISTEMA ELECTORAL}

A partir de la transformación del IFE en un órgano constitucional autónomo en 1996, el sistema electoral se fundamenta en dos elementos en constante equilibrio: la ciudadanización y los procesos técnicos. El primero es el área ciudadanizada, encabezada por un consejero presidente y ocho consejeros electorales nombrados con la aprobación de al menos dos terceras partes de los miembros presentes de la Cámara de Diputados, con perfiles ciudadanos independientes e imparciales, y con derecho a voto en el Consejo General, el máximo órgano de dirección. El segundo elemento es un cuerpo de funcionarios profesionales y especializados en aspectos técnicos necesarios para una elección. Estos funcionarios ocupan la Secretaría Ejecutiva del IFE y las direcciones ejecutivas de Registro Federal de Electores, Organización Electoral, Capacitación 
Electoral y Educación Cívica, Prerrogativas y Partidos Políticos, entre otras.

Este diseño dual se replica, con algunas diferencias, en casi todos los órganos decisorios del Instituto: Consejo General y Junta General Ejecutiva, consejos locales y juntas locales ejecutivas, consejos distritales y juntas distritales ejecutivas. En cada uno hay una presencia permanente de los representantes de los partidos políticos que tienen derecho a voz, pero no a voto. No sobra mencionar que en el caso del Consejo General y de los consejos locales y distritales quien preside el órgano de dirección se desempeña a la vez como cabeza de la rama ejecutiva. Entonces, el consejero presidente del Consejo General preside también la Junta General Ejecutiva y el presidente de un consejo local hace lo propio en la junta local ejecutiva, y lo mismo sucede a nivel distrital.

Este equilibrio entre las ramas ciudadanas y técnicas, que no está exento de una tensión permanente, logró que el IFE organizara las elecciones de 1997, 2000 y 2003 sin mayores cuestionamientos y con una legitimación reconocida tanto por los contendientes como por la opinión pública. La crisis institucional comenzó con el proceso electoral de 2006. Esa elección presidencial quedó ensombrecida y marcada por varios factores que erosionaron la confianza en el IFE. Los comicios fueron señalados por las intervenciones del presidente Vicente Fox a favor del candidato a la presidencia por el Partido Acción Nacional (PAN), Felipe Calderón Hinojosa, y contra el candidato de la Coalición por el Bien de Todos, Andrés Manuel López Obrador.

Otro factor determinante fue el estrechísimo margen de diferencia entre el primero y el segundo lugares, apenas de $0.56 \%$. Esta situación, referida por algunos como "la tormenta perfecta", fue la que detonó la decisión del Consejo General del IFE, que en voz de Luis Carlos Ugalde informó que no se darían a conocer los resultados del ejercicio estadístico de conteo rápido y que era necesario esperar a los cómputos definitivos del miércoles 9 de julio. Con esa decisión minimizó la importancia y trascendencia de las cifras que empezaba a arrojar el
Programa de Resultados Electorales Preliminares (PREP) durante la noche siguiente a la elección y, de paso, también la información que generon los 300 distritos electorales al hacer las sumas de absolutamente todas las actas de escrutinio y cómputo conforme se recibían los paquetes. De poco sirvió el trabajo técnico del Instituto, que no debemos escatimar en reconocer que fue igual que el de los procesos electorales anteriores, profesional y muy certero.

Las reacciones de los contendientes fueron inmediatas. El candidato de los partidos de izquierda cuestionó los resultados del PREP con el argumento de la presencia de un sinnúmero de inconsistencias en las actas y el famoso archivo de actas no publicadas. Aclaro que utilizó la palabra "publicadas" de manera deliberada, ya que los partidos políticos sí tuvieron acceso a esos archivos a través de sus representantes. Además de generar incertidumbre, lo comunicado por Luis Carlos Ugalde la noche del 6 de julio de 2006 evidenció la falta de decisión y de fuerza del Consejo General, el desperdicio y la mala utilización de los elementos técnicos que dan contención a los competidores durante las primeras horas después de la jornada electoral, es decir, los primeros resultados de la tendencia de la votación - conteo rápido y PREP-. ${ }^{1}$

El órgano máximo de dirección del IFE resentía las consecuencias del cambio de algunos de sus integrantes por parte de la Cámara de Diputados, mediante un procedimiento opaco y con una lógica de cuotas con afinidades partidistas, no con base en perfiles ciudadanos independientes. Hay que recordar que el Partido de la Revolución Democrática (PRD) no avaló la elección de los nueve integrantes con derecho a voto y que tanto el Partido Revolucionario Institucional (PRI) como el PAN tomaron la mala

\footnotetext{
${ }^{1}$ Los partidos habían acordado no difundir los resultados del conteo si la diferencia entre el primero y el segundo lugares era menor al margen de error del conteo, circunstancia que se cumplió. Sin embargo, se trataba de una decisión estratégica y delicada. Debió darse a conocer el resultado y explicarse a la ciudadanía claramente los márgenes de error de los tres análisis realizados por el comité de expertos.
} 
decisión de votar a los consejeros presidentes y a los ocho consejeros sin esperar el consenso con el PRD. En su momento, el proceso de renovación de integrantes fue muy criticado. Incluso, el Comité Conciudadano para la Observación Electoral - conformado por especialistas en materia electoral y organizaciones de la sociedad civil, que ha realizado observación electoral y análisis de las normas que rigen la materia con el ciudadano y sus derechos políticos como eje- lo calificó públicamente como un procedimiento lleno de tropiezos (CCOE, 2008).

En ese contexto surge la demanda lopezobradorista de "voto por voto, casilla por casilla". La respuesta del IFE ante la situación fue igualmente desafortunada, ya que desde la Dirección Jurídica se giró, vía fax, una instrucción a todos los consejos distritales del país para inhibir la apertura y recuento de los paquetes electorales durante las sesiones de cómputo el miércoles posterior a la jornada electoral de 2006. Finalmente, se realizó el recuento de 14\% de las casillas en los consejos distritales. El Tribunal los riesgos de una reforma modelada por los partidos políticos mayoritarios y a espaldas de la sociedad civil (CCOE, 2011: 190). De la reforma electoral de 2008 (Segob, 2008) sobresalen dos aspectos, que surgen como reacción a los sucesos de la elección presidencial de 2006: la inclusión de los recuentos totales en los consejos distritales del Instituto y las normas que buscaron equilibrar la contienda electoral - como el modelo de comunicación política en el que se prohibió la compra de espacios en radio y televisión y la limitación a la promoción personalizada de los funcionarios públicos, la regulación de las precampañas, la prohibición de la llamada "propaganda negra” y la figura del procedimiento sancionador electoral-.

Si bien hubo aciertos en la reforma electoral de 2008, no podemos dejar de lado que, como advirtió en su momento el Comité Conciudadano, se debilitó la parte ciudadanizada del Instituto. Hay tres ejemplos de esta situación: 1) la designación del contralor del IFE por la Cámara de Diputados y la creación de una unidad de fiscalización con autonomía técnica y de gestión, que invade la autonomía de la autoridad electoral; 2) la restricción en el número de comisiones en las que pueden participar los consejeros electorales, en las que se incorporó a representantes de los partidos políticos (CCOE, 2011: 191), y 3 ) la inclusión de los consejeros locales y distritales como sujetos de la Ley Federal de Responsabilidades de los Servidores Públicos. Respecto de la Contraloría Interna del IFE, nombrada por el Poder Legislativo, el Comité Conciudadano alertó desde septiembre de 2007:

consideramos que una Contraloría designada por los Partidos Políticos representados en el Congreso significará un foco de permanente tensión al interior de la institución y una amenaza a la autonomía. Por otra parte despojar a los consejeros electorales de la facultad de participar a través de una comisión específica en el análisis y deliberación de la forma en la que los partidos políticos hacen uso de los recursos, y crear un organismo prácticamente autónomo, limitado a realizar auditorías que sólo conducirán a

\footnotetext{
${ }^{2}$ Como lo expresa Alberto Olvera: "la mucha tinta que se ha utilizado en nuestro país acerca de nuestra larga transición a la democracia, caracterizada por construir en una serie muy prolongada y aún inacabada de reformas electorales, magnificó el protagonismo de los partidos políticos en el proceso" (Olvera, 2008: 46).
} 
observaciones generales sobre el estado de las finanzas de los partidos, debilita el carácter ciudadano de la vigilancia del ejercicio de recursos públicos de los partidos. Ello constituye un retroceso de la mayor gravedad (CCOE, 2011: 191-192).

$\mathrm{Al}$ analizar temas de rendición de cuentas de los órganos autónomos es pertinente aclarar que de ninguna manera se justifica que no rindan cuentas a cabalidad ni exentarlos del cumplimiento de la ley. Simplemente, los regímenes de responsabilidades para este tipo de órganos de Estado deben garantizar su autonomía e independencia, situación que no es clara respecto de la Contraloría del IFE. Como ya se dijo, después de la reforma de 2008 los consejeros locales y distritales son sujetos de la Ley Federal de Responsabilidades de los Servidores Públicos sin tener atribuciones en lo individual, o sea, fuera del órgano colegiado, y también sin tener una relación laboral con el IFE, lo que minimiza su papel de ciudadanos externos y los convierte en una suerte de empleados de segunda.

La preocupación de que los consejeros locales y distritales cumplieran con las leyes electorales fue el argumento para incluirlos como sujetos obligados del régimen de responsabilidades, con lo que se desvirtúa su participación como agentes independientes y externos a la institución. Veamos algunos ejemplos: si un consejero de algún consejo local pretendiera detener la votación en una de las casillas de su entidad, el presidente de la casilla correspondiente tendría que solicitar el apoyo de la fuerza pública para restaurar la operación de la misma, al igual que lo hubiera hecho en caso de tratarse de cualquier otra persona. En realidad, no se requiere de la aplicación de la Ley de Responsabilidades de los Servidores Públicos para obligar a todas las personas involucradas en un proceso electoral a respetar las leyes electorales. Lo anterior cobra fuerza si se considera que los consejeros electorales locales y distritales no cuentan con atribuciones en lo individual, sino únicamente como cuerpo colegiado. Por ello si alguna de sus decisiones no se apega a la ley electoral, seguramente ésta no será acompañada por sus colegas, y si fuera una posición mayoritaria la contraria a la ley los representantes de partido podrían recurrirla ante la instancia superior o, en su caso, ante el Trife. Llevado el caso al absurdo, sería como si los funcionarios de mesas directivas de casilla quedaran sometidos al régimen de responsabilidades por las labores que desempeñan el día de la jornada electoral. Pongamos el caso de que un funcionario de mesa directiva de casilla venda las boletas o decida llevarse el paquete a su casa la noche de la elección. Estaría cometiendo un ilícito electoral y sería juzgado por ello, sin que sea necesario inhabilitarlo del servicio público con base en la Ley Federal de Responsabilidades de los Servidores Públicos. Lo anterior no tiene sentido porque precisamente lo que legitima su actuación es su carácter de ciudadano, no su investidura en autoridad electoral. Lo mismo puede aplicarse a los consejeros locales y distritales. Por eso no reciben un salario, sino una dieta de asistencia. Otro golpe que debilitó la cara ciudadana de la institución fue el retraso de un año para nombrar a tres de los consejeros electorales en que incurrió la Cámara de Diputados, lo que dejó incompleto al órgano máximo de decisión del IFE todavía en el inicio del Proceso Electoral Federal 2011-2012. Si bien se nombró a los tres consejeros vacantes con un consenso de todas las fuerzas políticas, el daño ya estaba hecho.

Una vez fijados los antecedentes y el contexto resulta pertinente agregar que la reforma de $2008 \mathrm{im}$ puso muchos retos al IFE. Casi podríamos pensar que muchas de las disposiciones parecían imposibles de cumplir: por ejemplo, los monitoreos a los medios de comunicación, la fuerte carga litigiosa con los procedimientos especiales sancionadores y las quejas, y por supuesto los recuentos de votos. Parecían estar diseñadas para reventar a la institución. En realidad, cumplir con ellas condujo a la institución al límite de sus recursos humanos y técnicos, lo que se agudizó en los consejos distritales, por ser los órganos competentes para resolver los procedimientos especiales sancionadores y las quejas y realizar los recuentos. Todos los temas mencionados son relevantes, pero para la ciudadanía en 
general en este Proceso Electoral Federal 2011-2012 el IFE se enfrentó a dos pruebas de fuego: la fiscalización de los recursos erogados en las campañas - que en razón de no haberse concluido trataré sólo de manera parcial-y los recuentos - de los que haré un análisis más detallado por haberse llevado a cabo-.

\section{LA FISCALIZACIÓN DE LOS GASTOS DE CAMPAÑA}

El 16 de mayo de 2012, el Consejo General del IFE aprobó un procedimiento de fiscalización anticipada - como consecuencia de una iniciativa del PRD-, que consistió en que la Unidad Técnica de Fiscalización adelantara cinco meses la entrega del Dictamen Consolidado y Proyecto de Resolución de los informes de campaña de los candidatos a la presidencia de la república (IFE, 2012b). Como resultado, de manera inédita, se generó una escisión desarrolladas para obtener los cargos de presidente, diputado y senador, con la finalidad de conocer con anticipación los gastos erogados para las campañas presidenciales. Así, se emitió el Dictamen Consolidado de las irregularidades encontradas por separado en las campañas de senadores y diputados. En cumplimiento de este acuerdo, dentro de la orden del día de la sesión extraordinaria del Consejo General del IFE del 30 enero de 2013 se presentó el Dictamen Consolidado de los Recursos de los Partidos Políticos y Proyecto de Resolución del Consejo General del Instituto Federal Electoral respecto de las irregularidades encontradas en el Dictamen Consolidado de la revisión anticipada de los Informes de Ingresos y Gastos de Campaña, relativos a los candidatos a la presidencia de los Estados Unidos Mexicanos para el Proceso Electoral Federal 2011-2012. Una mayoría de siete consejeros electorales solicitaron el retiro de dicho punto de la orden del día para contar con más tiempo para el análisis minucioso de dicho dictamen (IFE, 2013c: 56).

Como consecuencia de la reforma electoral de 2008, la Unidad de Fiscalización ejerció su recién otorgada autonomía técnica y giró el expediente en cuestión a los consejeros electorales sólo tres días antes de la sesión en la que se votaría su aprobación, sin haberse discutido en comisión. Dado el retiro del punto en la sesión anterior, el 6 de febrero de 2013 se sometió a la consideración del Consejo General el Dictamen Consolidado de las irregularidades encontradas a los gastos de campaña para presidente de la república y fue rechazado por cinco votos en contra y tres a favor. Con una mayoría de cinco votos, se decidió su devolución a la Unidad Técnica de Fiscalización para "incorporar la definición del concepto de campaña beneficiada, a efecto de motivar adecuadamente los elementos y razones para acreditar el beneficio de las campañas relacionadas con una misma erogación" (IFE, 2013b: 6) y "para realizar un análisis con los informes de diputados y senadores" (IFE, 2013d).

Estas decisiones se tomaron sólo dos semanas después de que se exonerara al PRI por una mayoría de cinco votos contra cuatro - con un desempate del consejero electoral Sergio García Ramírez- de su responsabilidad en una queja sobre financiamiento de ente prohibido, mejor conocida como "Caso Monex" (IFE, 2013a). A este respecto, el Consejo General del IFE dictaminó que no hubo responsabilidad de los partidos políticos Revolucionario Institucional y Verde Ecologista de México por financiamiento ilegal, por origen o destino de los recursos involucrados en esta queja para las campañas de la Coalición Compromiso por México. La única consecuencia fue que los montos detectados - poco más de 50.5 millones de pesos - deben ser agregados a los gastos de campaña para efecto de determinar los rebases de topes fijados a las mismas.

El artículo 342, inciso $f$, del Código Federal de Instituciones y Procedimientos Electorales (Cofipe) estipula como una infracción de los partidos políticos exceder los topes de campaña. Lo mismo se establece en el artículo 344 para precandidatos o candidatos a cargos de elección popular. La deficiencia del sistema 
consiste en que se señalan sanciones específicas como consecuencia a las infracciones cometidas para los partidos políticos y también para precandidatos o candidatos en el artículo 354 del mismo ordenamiento. Pero al momento de determinar los topes de gastos de campaña, los únicos sujetos imputables que mantienen su personalidad son los partidos políticos. En los plazos de entrega y revisión de los gastos de campaña, y la determinación del rebase de topes, si los hubiera, los contendientes ya perdieron su calidad de candidatos, por lo que no son sancionables de conformidad con las normas electorales. Cabe recordar que la aplicación de sanciones debe seguir un régimen de aplicación estricta. La situación actual prevista en la ley es: aunque se actualice el rebase de topes de campaña, el candidato electo o el funcionario público no sufrirán consecuencia jurídica alguna, y el partido político que los haya postulado recibe la única sanción prevista. Este estatus legal prevalece incluso después de los multicitados casos "Pemexgate" y "Amigos de Fox" (Córdova y Murayama, 2006). Si se analiza el costo-beneficio de su cumplimiento, con el régimen de sanciones citado las normas se tornan funcionales a los intereses partidistas.

\section{EL VOTO POR VOTO}

Según la redacción vigente del Cofipe, bajo ciertos supuestos específicos, los consejos distritales deben realizar un nuevo escrutinio y cómputo de las casillas cuando existan errores o inconsistencias evidentes que no se puedan aclarar con otros elementos a satisfacción plena del representante partidista que lo solicite. La petición puede hacerse cuando la cantidad de votos nulos supere la diferencia entre el primero y el segundo lugares de votación y cuando

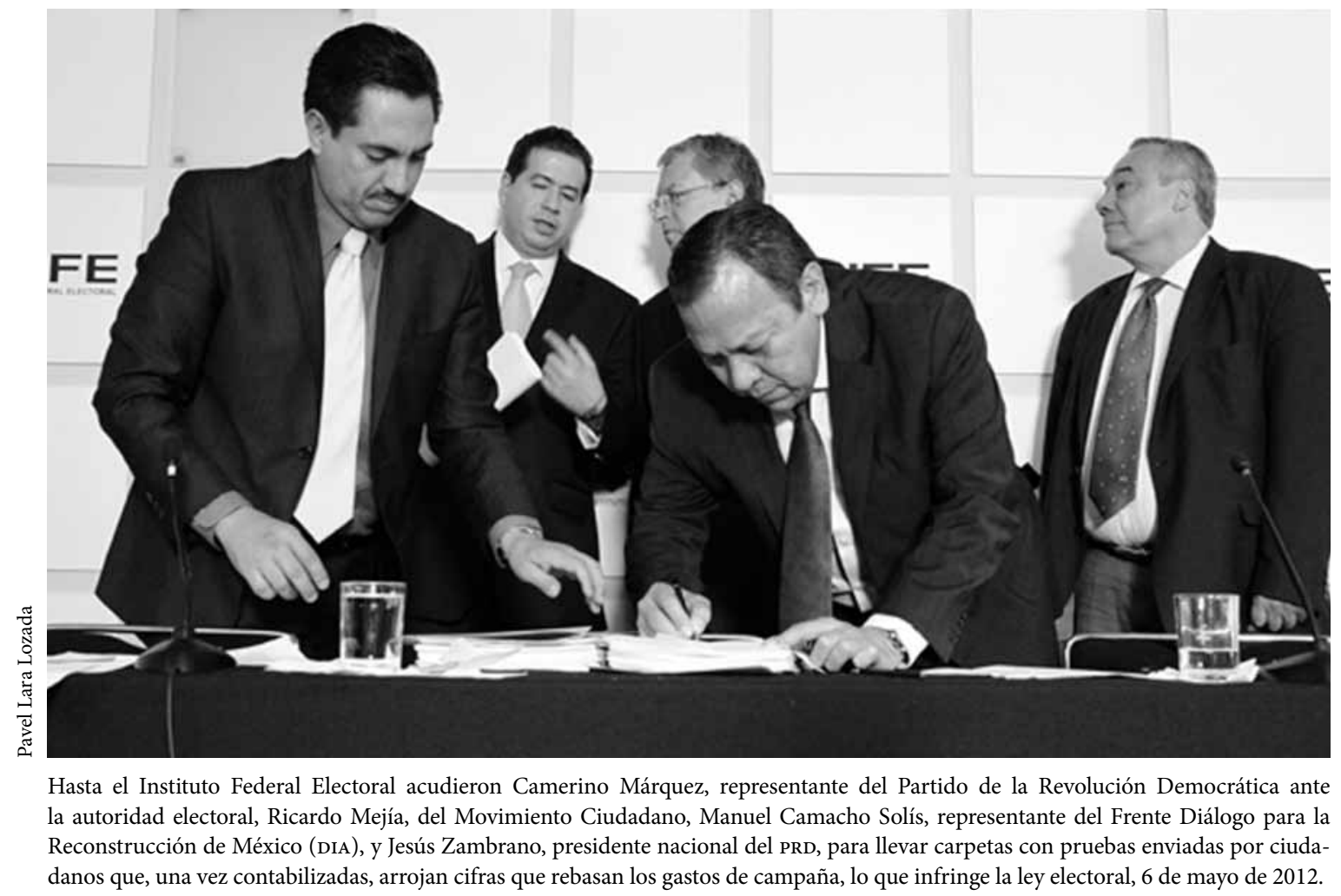


todos los votos se hayan emitido a favor de un mismo partido (Cámara de Diputados, 2008b: art. 295, $\$ 1$, incisos $d$ y $b$ ). Para la realización del recuento total en un distrito, el Cofipe también prevé que se cumpla la condición de una diferencia menor o igual a un punto porcentual y que este procedimiento haya sido solicitado por el representante del partido político posicionado en segundo lugar (Cámara de Diputados, 2008b: art. 295, \$2, 3 y 4). Este diseño es deficiente por dos razones: se trata de una norma pensada en función de los intereses de los partidos políticos y no de darle certeza a la ciudadanía sobre la elección, además de que no resuelve el problema para el que se instituyó.

Es importante reflexionar en torno a cuál es el propósito real que persigue la norma. Pudiera parecer que más que a abonar a la certeza, se le apuesta a la posibilidad de corregir los errores humanos en el cómputo de votos, o eso hace suponer la exigencia de que la diferencia deba ser menor o igual a $1 \%$. Se trata de un supuesto que surtirá efecto en los casos en que, en efecto, el resultado de la elección en un distrito determinado pueda revertirse. Por eso se otorga la facultad de solicitar el recuento sólo al partido o coalición del candidato o candidata que se encuentre en segundo lugar. No puede realizarse de oficio por la autoridad electoral ni ser solicitada por la ciudadanía. El modelo tiene como consecuencia la posibilidad real de revertir un resultado electoral al corregir los errores del cómputo de votos en casilla, por lo que establece como presupuesto una diferencia numérica mínima. Así, resulta atractivo para el partido político que está en segundo lugar por la posibilidad real de lograr el triunfo y para el competidor en primer lugar porque puede tener un triunfo legitimado por un recuento.

Si con los recuentos se buscara dotar de certeza a la elección, entonces los supuestos podrían ser distintos, como el desempeño de los funcionarios de casilla, la compra y coacción del voto, situaciones registradas en las actas de incidentes o incluso la labor de algunos miembros del servicio profesional electoral o de integrantes de consejos distritales, la presencia desequilibrada de los contendientes en los medios de comunicación, etc. La realidad es que el legislador limitó los casos en los que se puede practicar un recuento total. Ahora bien, la Ley General del Sistema de Medios de Impugnación en Materia Electoral contempla la posibilidad de solicitar la nulidad de la votación recibida en casilla por situaciones como dolo o error en el cómputo de votos, de nuevo si la votación fuera determinante para el resultado de la elección, por haber permitido que votaran personas sin credencial o haber impedido el acceso a los representantes de partido (Cámara de Diputados, 2008a: art. 75, incisos $f, g$ y $h$ ). Ahora, el recuento está previsto en la ley con la casilla o bien el distrito como unidad, no para el resultado global de la elección (Cámara de Diputados, 2008b: arts. 295, 297 y 298). El diseño es curioso, porque en México las elecciones no se ganan con base en un agregado del número de casillas ganadas ni de distritos ganados, sino con la sumatoria total de los votos obtenidos, en caso extremo, hasta por un voto de diferencia.

$\mathrm{Al}$ respecto, es interesante mencionar el caso de empate que se actualizó en la elección de regidores en el municipio de Mama, Yucatán, en el que después del estrecho margen de diferencia entre el primero y el segundo lugares se realizó el recuento en el Consejo General del Instituto de Procedimientos Electorales y Participación Ciudadana (Ipepac) del Estado de Yucatán. Antes de aprobar el recuento total de la elección, uno de los consejeros manifiesta:

\footnotetext{
nosotros estamos tratando, en estos actos que hoy se programan, en tratar de hacer prevalecer la voluntad de los ciudadanos de Mama; nosotros en responsabilidad recibimos los paquetes electorales, que de manera desesperada, creo que hasta aterrada, los consejeros trajeron y a lo mejor hasta perseguidos llegaron a la ciudad de Mérida, lo que hoy queremos hacer es reponer todo esto y tratar de corregir y limpiar, en este caso la elección (Ipepac, 2012: 8).
}

Como vemos, el consejero interpreta el valor de la norma, el valor no escrito protegido por la disposición legal. Para él, desde una óptica ciudadana, es la 
certeza en la elección. Finalmente, el Consejo General del Ipepac decidió de manera unánime conceder la petición del Consejo Municipal y realizar el recuento. Al terminar el nuevo escrutinio y cómputo se registró un empate con 927 votos para cada una de las fuerzas políticas, PAN y PRI-PVEM, por lo que el Consejo General procedió a su declaración formal y notificó al Congreso del Estado para los efectos a que hubiera lugar. Esta decisión fue impugnada por el PAN ante el Tribunal de Justicia Electoral y Administrativa del Estado de Yucatán (Teeyuc) y confirmada el 11 de agosto de 2012 (Teeyuc, 2012). Ahora bien, en la impugnación ante el Teeyuc el pAn buscaba anular una casilla, todos los votos recibidos en la casilla 234 contigua 1, con el argumento de un cambio de domicilio injustificado.

Esto es relevante porque el partido que impugna busca que se anule una casilla para cancelar el empate declarado después del recuento total de votos. $\mathrm{Al}$ anular una casilla, se reajusta el cómputo total de la elección y, para efectos prácticos, hay un desempate. No sobra recordar, como constatan las actas del Consejo General del Ipepac, que el recuento en el Consejo Municipal se llevó a cabo entre mucha tensión y riesgos para los involucrados, por lo que trasladaron la totalidad de paquetes a la autoridad máxima estatal. Este ejemplo pone de manifiesto dos situaciones: 1) que en el sistema electoral mexicano la elección se gana incluso por un voto, y 2) que las normas electorales están diseñadas de acuerdo con el interés de los partidos, más que con el de los ciudadanos - idea que desarrollaré más adelanteEn este caso, si el Teeyuc hubiera anulado la casilla impugnada, la voluntad ciudadana verificada en un recuento "voto por voto" quedaría anulada con base en un tecnicismo legal. Por fortuna, eso no sucedió y el Tribunal confirmó el empate declarado por el Consejo General del Ipepac.

Ahora bien, en el Distrito 06 del Estado de México, en Coacalco de Berriozábal, después de un recuento total para la elección de diputado, el Consejo Distrital del IFE entregó la constancia de mayoría al candidato de la Coalición Movimiento
Progresista - PRD-PT-MC-, quien obtuvo una ventaja de 194 votos sobre el candidato de la Coalición Compromiso por México - PRI-PVEM-, que impugnó la decisión ante la Sala Regional del Trife, correspondiente a la Quinta Circunscripción Plurinominal, con sede en Toluca, Estado de México. Por unanimidad de votos, la Sala Regional determinó la anulación de 13 casillas, con base en dos causales: 1) la recepción de votos de personas no idóneas, básicamente, que los funcionarios de mesas directivas de casilla no pertenecían a esa sección, y 2) que algunos funcionarios de casilla se desempeñaban como funcionarios públicos a nivel municipal, por lo que se vio afectada la libertad de los electores. Finalmente, el candidato de Compromiso por México obtuvo el primer lugar con una diferencia de 311 votos sobre el candidato de Movimiento Progresista (Trife, 2012a). Esta sentencia es recurrida ante la Sala Superior, que confirma, también por unanimidad de votos, la sentencia de la Sala Regional Toluca (Trife, 2012b). El asunto captó la atención de la prensa porque esta decisión cambió el equilibrio en el Congreso. Entre el PRI, el PVEm y el Partido Nueva Alianza (Panal), con los que el primero ha tenido acuerdos, suman 251 diputados, de manera que podrían conseguir una mayoría simple en la Cámara de Diputados (Taniguchi, 2012).

Como se muestra en estos ejemplos, el hecho de que los partidos políticos puedan impugnar casillas de manera aislada para buscar su invalidación -después de un recuento total realizado por consejeros electorales distritales y miembros del servicio profesional electoral, utilizar al límite todos sus recursos técnicos y humanos y contener el conflicto entre los partidos y en ocasiones entre la población- resulta inútil si un tribunal revierte el resultado y anula algunas casillas por un tecnicismo jurídico. En todo caso, la posibilidad de impugnar casillas en lo individual debería otorgarse a los partidos antes de la realización del recuento total, o incluso establecer que el ejercicio de uno de esos derechos anulara al otro, como quien se somete a un arbitraje, que renuncia al derecho de utilizar otra vía 


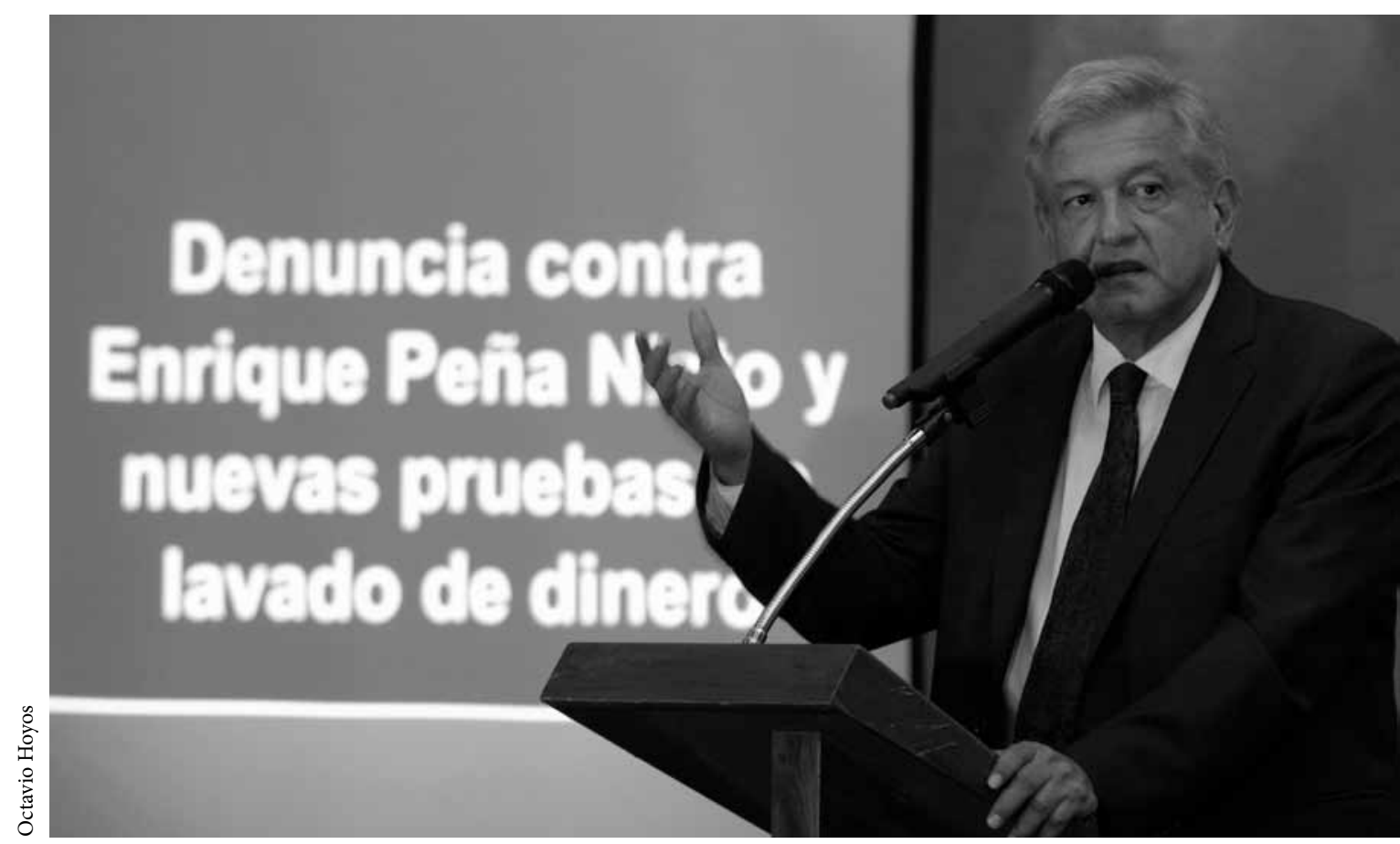

Andrés Manuel López Obrador, candidato de las izquierdas a la presidencia de la república, durante una conferencia de prensa sobre el caso de tarjetas Monex, Distrito Federal, 18 de julio de 2012.

que la judicial para resolver ese conflicto. En realidad el sistema termina por favorecer estrategias de litigio diseñadas por los partidos políticos que no guardan ninguna relación con la voluntad ciudadana. ${ }^{3}$

La otra deficiencia de la norma es que la hipótesis del recuento total del artículo 295 del Cofipe resulta funcional sólo para el caso de la elección de diputados federales - la insuficiencia del modelo legal se ilustra en el siguiente ejemplo-. Esta falla fue

\footnotetext{
${ }^{3}$ Vale la pena revisar el caso de la Presidencia Municipal de Netzahualcóyotl, en el que se suspendió el recuento en la junta distrital y se enviaron paquetes al Instituto Electoral del Estado de México, cuyo Consejo revirtió la elección a favor del PRD y se impugnó. El Tribunal Electoral del Estado de México aún no falla a este respecto. También está el caso del Distrito 12 de Ixtapaluca, donde el PRI quedó en segundo lugar para la elección presidencial, solicitó la apertura de 30 paquetes y después del recuento subió al primer escaño. El PRD quedó en segundo lugar y desde esta posición pidió el recuento total de la elección presidencial. Como se observa, todo depende de la estrategia de cada partido, no es un mecanismo que dé certeza a los ciudadanos.
}

esgrimida por el PAN ante la Sala Regional del Tribunal Electoral con sede en Monterrey, Nuevo León, frente a la diferencia de $0.58 \%$ entre su candidato a senador por mayoría relativa y el del PRI. Después de hacer una interpretación funcional de la norma contenida en el artículo 295, la Sala Regional concede un recuento total para la entidad y ordena su realización a los Consejos Distritales del IFE (Trife, 2012c):

este órgano de justicia comicial estima que la negativa recaída a la solicitud de recuento aludida, se basa en una premisa contraria a la naturaleza del sistema normativo que prevé el recuento total en la elección precisada, tornándolo infuncional.

En efecto, esto es, la consecuencia de aplicar de manera letrística las normas atinentes llevaría al extremo de que resultase intrascendente que exista una diferencia igual o menor a un punto porcentual entre los contendientes que ocupan el primer y segundo lugar de la elección de senador (Trife, 2012c: 39). 
Cabe aclarar que el único distrito que cayó en el supuesto de la diferencia numérica fue el que tiene cabecera en Guadalupe, con una diferencia de $0.86 \%$ del PAN sobre el PRI. Éste tendría que solicitar el recuento porque está en el supuesto de aplicación estricta del artículo 295, situación contraria a sus intereses en cuanto a la elección total en el estado. Más tarde esta decisión es recurrida ante la Sala Superior por el PRI, bajo la exigencia de una interpretación literal de la norma. Con base en una interpretación "auténtica" de las normas contenidas en el Cofipe, la Sala Superior del Trife revocó la resolución de la Sala Regional y aplicó la norma de manera literal sin dar cabida a la duda razonable del error humano, que puede suceder al tener una diferencia mínima en el resultado total para la elección (Trife, 2012e).

Además de ilustrar la insuficiencia de la norma, este par de decisiones nos dan la oportunidad de contrastar dos razonamientos jurisdiccionales. La Sala Regional que interpreta la norma de una manera sistemática y funcional, y responde a la necesidad de dotar de certeza a una elección al corregir posibles errores humanos del cómputo ante un margen reducido de diferencia entre el primero y segundo lugares. A diferencia de los casos para la elección presidencial o de senadores, el supuesto de la ley para la realización de los recuentos funciona para el caso de la elección de diputados, porque se realiza con base en la votación total de un distrito electoral uninominal. Si la diferencia es igual o menor a $1 \%$, o incluso si los votos nulos superan la diferencia entre el primero y el segundo lugares - como de hecho ocurrió en la elección de 2006-, la norma prevista en el Código para el recuento seguramente no hubiera sido suficiente para generar la convicción en todos los actores, si se hubiera aplicado como dispuso la Sala Superior del Trife. Entonces, aunque se hubiera presentado un escenario similar al de 2006 entre Enrique Peña Nieto y Andrés Manuel López Obrador, el recuento hubiera ocurrido como en efecto fue: tomando como base las casillas y los distritos reñidos, cuando lo que genera la diferencia menor a $1 \%$ fuera la suma global.
Una vez analizadas distintas situaciones de recuentos totales en los distritos, que proporcionaron los presupuestos para actualizar la hipótesis legal, podemos examinar la solicitud de apertura de paquetes formulada por la Coalición Movimiento Progresista, integrada por los partidos de la Revolución Democrática, del Trabajo y Movimiento Ciudadano, el 3 de julio de 2012, y la atención que recibe del Consejo General del IFE. Ricardo Monreal Ávila, en su carácter de coordinador y representante de Andrés Manuel López Obrador, con Jesús Zambrano, como presidente nacional del PRD, Jaime Cárdenas Gracia, asesor jurídico, y los representantes ante el Consejo General del IFE de los partidos que conformaron la coalición y su candidato a la presidencia solicitaron durante la sesión permanente del Consejo General, con base en las fracciones 1, 2, 3 y 4 del artículo 295 del Cofipe, la realización de un nuevo escrutinio y cómputo de la votación recibida en las 143132 mesas directivas de casilla. A la petición se anexó un escrito en el que se exponen situaciones, al parecer reportadas en las casillas, como la presencia de personas sospechosas, que la tinta indeleble se borra, que personas de alguna fuerza política apuntan a los votantes, que se compran votos, etc. Sin embargo, después de analizar cuidadosamente el documento no se advierte que se acrediten los supuestos que se refieren a errores o inconsistencias de las actas, número de votos nulos mayor que la diferencia entre el primero y segundo lugares o bien que todos los votos hayan sido emitidos a favor de un mismo candidato. No hubo ningún tipo de resultados electorales en dicho escrito. Como respuesta a la solicitud de la Coalición Movimiento Progresista, el Consejo General en la sesión extraordinaria del 3 de julio de 2011 emite un acuerdo (IFE, 2012d). En el segundo punto el Consejo General:

reconoce la relevancia jurídica de las sesiones de cómputos distritales en tanto que su objetivo no sólo es el de realizar las sumas precisas de los resultados de las actas que consignan la votación emitida 
en los respectivos distritos para las elecciones de Presidente, Senadores y Diputados, sino también el de generar la mayor certeza posible de que los votos emitidos por los ciudadanos serán escrupulosamente respetados y contados en un ejercicio público ante la presencia de los representantes de los partidos políticos en los consejos distritales (IFE, 2012d: 10).

En el punto cuarto del acuerdo, la autoridad electoral dispuso:

en la aplicación del referido artículo 295, que realicen los Consejos Distritales durante el proceso de cómputo distrital, garantizarán el principio de certeza establecido por el artículo 41 de la Constitución Política de los Estados Unidos Mexicanos, privilegiando para ello el criterio interpretativo que busque en todo momento la satisfacción plena de quien lo haya solicitado (IFE, 2012d).

Esta respuesta, que es una reacción muy diferente a la instrucción girada desde las oficinas centrales del IFE en el proceso electoral de 2006 para inhibir la apertura de paquetes, deja ver que la norma debe aplicarse firmemente por los Consejos Distritales, con el objetivo de alcanzar la plena satisfacción de quien lo solicite. El mensaje fue recontar los paquetes conforme a las hipótesis legales. Aunque este acuerdo se tomó después de la jornada electoral, es importante señalar que desde el 25 de abril de 2012 el Consejo General aprobó un acuerdo en que se emitieron los lineamientos para el desarrollo de la sesión de cómputo distrital. Con éstos se buscó que los 300 Consejos Distritales del Instituto pudieran hacer frente incluso a un recuento total de paquetes electorales. Las personas responsables de dichos conteos serían los consejeros distritales y los miembros del servicio profesional electoral, auxiliados por los capacitadores asistentes.

En los lineamientos se especificó cómo funcionarían los grupos de trabajo y los representantes de partidos que podrían estar presentes para observar el procedimiento. Esta previsión tomó en cuenta incluso las limitaciones en los espacios físicos de las oficinas del Instituto, el relevo de los consejeros y vocales, las necesidades logísticas, como carpas o puntos de red. Como material de apoyo, el Consejo General aprobó también desde el 7 de junio de 2012 un cuadernillo de consulta para votos válidos y votos nulos para el desarrollo de la sesión especial de cómputos distritales (IFE, 2012c). La idea era tener una guía al momento de la realización de los cómputos distritales para agilizar las discusiones sobre los votos emitidos de manera inusual. En dicho material se incorporó la importancia de desentrañar la intención del votante en cada boleta electoral. Se diseñaron e impartieron capacitaciones específicas para todas las personas involucradas. Con estas previsiones, el Consejo General había mandado el mensaje correcto a los Consejos Distritales. Ahora bien, el porcentaje de paquetes recontados en cada distrito en cada una de las tres elecciones federales anteriores tuvo comportamientos muy diversos, como puede observarse en la gráfica 1. Finalmente, podemos decir que el IFE hizo frente al reto que implicaban los recuentos de manera profesional. Se recontaron 78469 paquetes $-54.82 \%$ - para la elección de presidente, $81655-57.05 \%$ - para la de diputados y $82288-57.49 \%$ - para la de senadores a nivel nacional. En esta elección, con todo y las deficiencias de la norma, resultó suficiente el recuento realizado por el IFE, incluso la impugnación sobre la elección interpuesta por el Movimiento Progresista no versaba de manera central sobre este asunto. Con todo lo anterior, los partidos impugnaron 296 de los 300 cómputos distritales para la elección de presidente. Se modificaron los resultados de 56 distritos después de que el Trife ordenara nuevos escrutinios y cómputos. Merece un análisis más detallado conocer el tipo de interpretaciones que se hicieron al artículo 295 del Cofipe, según los índices de paquetes electorales recontados, para contrastar con el tipo de resultados electorales según se encuadraran en las hipótesis por las que se pueden abrir los paquetes. 
Gráfica 1. Cantidad de distritos electorales según el porcentaje de apertura de paquetes

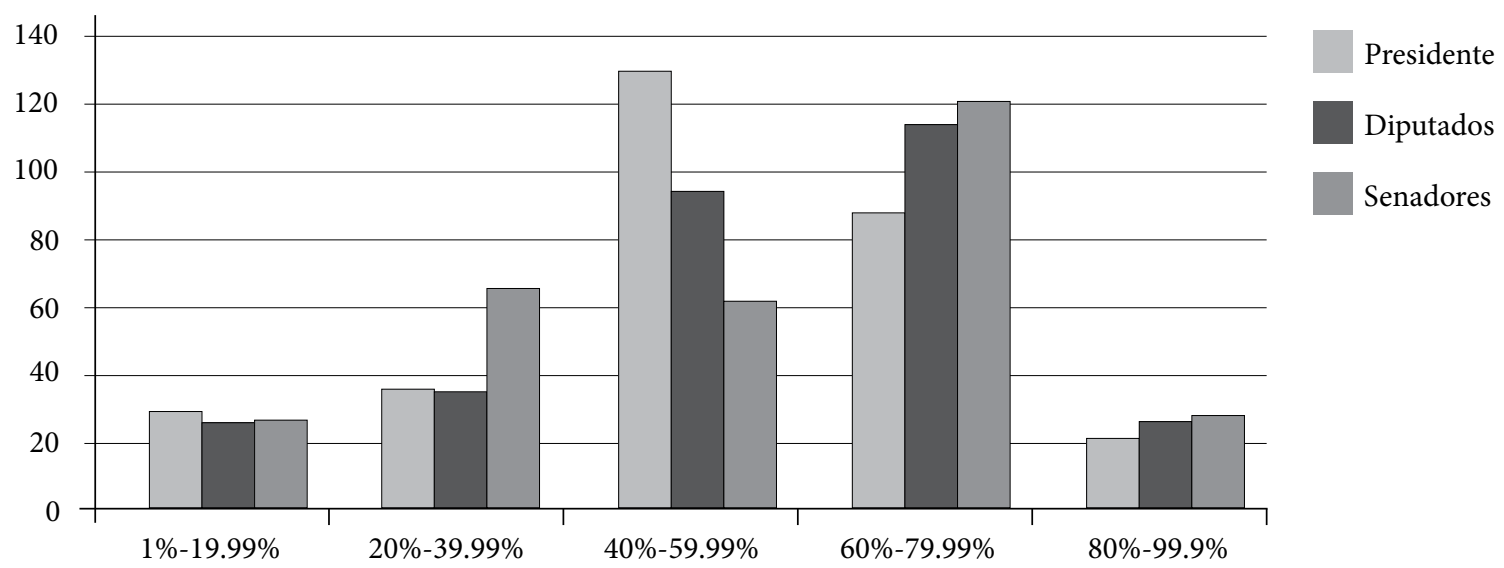

Fuente: Elaboración propia.

\section{CONCLUSIONES}

La primera prueba de fuego de la elección está aún sin resolver: la fiscalización. El proceso de investigación a cargo de la Unidad de Fiscalización es exhaustivo y minucioso y deberá concluir en los meses por venir. Seguramente, el IFE entregará una investigación completa y profesional. El problema será, de nuevo, el alcance de las normas. Por más que se compruebe el rebase de los topes de campaña y el financiamiento ilegal, la consecuencia será la imposición de multas a los partidos políticos infractores. Además, cabe mencionar que "para que una conducta sea reincidente a los ojos de nuestras autoridades electorales, es necesario que ésta se refiera exactamente a los mismos hechos" (Pérez, 2010: 147). Las multas que se impongan serán proporcionales a las violaciones que se detecten, tal vez de varios millones de pesos, pero se pagarán con el financiamiento público que reciben los partidos políticos, es decir, con nuestros impuestos. Se trata de un sistema de normas en el que las sanciones impuestas por los actos ilegales no alcanzan a los responsables, que muchas veces ejercen un cargo público de elección popular. Es un sistema que a los ojos de la ciudadanía deja impunes a quienes violan las normas del financiamiento, que buscan lograr la equidad en las contiendas electorales.

La segunda prueba de fuego, el recuento de los paquetes electorales, quedó superada para esta elección presidencial. La diferencia de más de tres millones entre el primero y segundo lugares contribuyó en buena medida a que la norma funcionara a pesar de las limitaciones que tiene el recuento total para la elección de senadores y presidente de la república, sin perder de vista que la norma le es funcional a los partidos políticos más que a la ciudadanía, ya que son los únicos facultados para solicitar su aplicación. Sin duda se requieren cambios, pero debemos cuidar que no haya retrocesos en el sistema electoral. No podemos regresar a un padrón no confiable, a cuando no se sabía realmente si las cifras reflejadas en los resultados del PREP o de los cómputos estaban respaldadas con votos en cada uno de los paquetes electorales, a la compra directa de tiempo en radio y televisión por los partidos políticos, a cuando se desconocía quiénes eran los funcionarios de mesas directivas de casilla. En el análisis de la aplicación de dos grupos de normas clave del sistema electoral vemos cómo las autoridades electorales tienen frente a sí a dos destinatarios claramente identificables: los partidos políticos y la ciudadanía. 


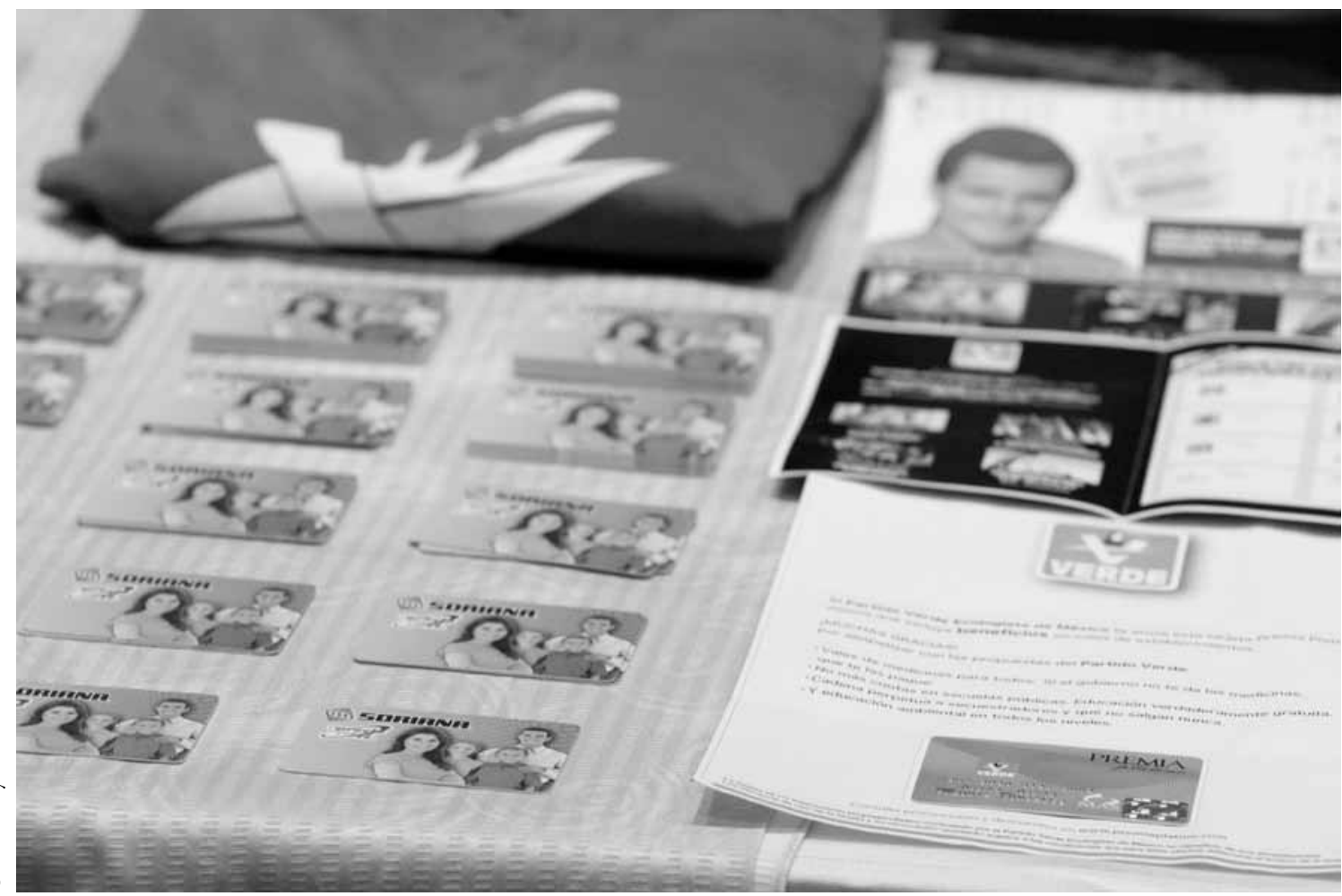

Conferencia y presentación de cientos de tarjetas de la tienda departamental Soriana por parte del candidato del PRD, Andrés Manuel López Obrador, 12 de julio de 2012.

Estas dos caras de la moneda no siempre responden a los mismos intereses o motivaciones. Los ajustes a las normas electorales se harán más evidentes en un futuro próximo, pero si deseamos una autoridad electoral sólida y confiable, las modificaciones deben realizarse en función de la ciudadanía, tomando como eje el interés público, y no el de los partidos políticos.

\section{BIBLIOGRAFÍA}

Alcocer, Jorge y Lorenzo Córdova, 2010, Democracia y reglas del juego, Instituto de Investigaciones Jurídicas, Universidad Nacional Autónoma de México, México.

Aziz Nassif, Alberto, 2008, "La pieza que faltaba", en Desacatos. Revista de Antropología Social, núm. 28, pp. 196-199.
Cámara de Diputados del H. Congreso de la Unión, 2008a, Ley General del Sistema de Medios de Impugnación en Materia Electoral, Cámara de Diputados, en línea: <http://www.diputados.gob.mx/LeyesBiblio/pdf/ 149.pdf $>$.

-, 2008b, Código Federal de Instituciones y Procedimientos Electorales, Cámara de Diputados, en línea: $<$ http://www.diputados.gob.mx/LeyesBiblio/pdf/COFIPE.pdf $>$.

—_ 2013a, Código Penal Federal, Cámara de Diputados, en línea: <http://www.diputados.gob.mx/LeyesBiblio/pdf/9.pdf>.

- 2013b, Constitución Política de los Estados Unidos Mexicanos, Cámara de Diputados, en línea: <http:// www.diputados.gob.mx/LeyesBiblio/pdf/1.pdf >.

Castillo González, Leonel, 2006, Derecho electoral, Tribunal Electoral del Poder Judicial de la Federación, México.

Centro de Estudios Sociales y de Opinión Pública de la Cámara de Diputados (CESOP), 2003, "El IFE en la opinión pública”, Centro de Estudios Sociales y de 
Opinión Pública de la Cámara de Diputados, en línea: $<$ http://www.diputados.gob.mx/cesop/doctos/ife_oppu.pdf $>$.

Comité Conciudadano para la Observación Electoral (CCOE), 2008, "Boletín de prensa", 22 de enero, en línea: $<$ http://comiteconciudadano.googlepages.com/ Comunicado_23enero2008.pdf $>$.

, 2011, "Riesgos de una reforma a modo de los partidos mayoritarios y a espaldas de la sociedad civil", en Iniciativa Ciudadana y Desarrollo Social/Comité Conciudadano para la Observación Electoral, Observación de las instituciones electorales en el proceso 20082009. Consejo General del IFE y Tribunal Electoral del Poder Judicial de la Federación, Iniciativa Ciudadana y Desarrollo Social, Comité Conciudadano para la Observación Electoral, México.

Consulta Mitofsky, 2012, "México: confianza en las instituciones", 7 de octubre, en línea: <http://consulta.mx/ web/images/mexicoopinapdf/20120830_NA_Confianza_Instituciones.pdf $>$, pp. 3-5.

Córdova, Lorenzo y Ciro Murayama, 2006, Elecciones, dinero y corrupción. Pemexgate y Amigos de Fox, Cal y Arena, México.

Crespo, José Antonio, 2008, 2006: hablan las actas, Random House Mondadori, México.

Instituto de Procedimientos Electorales y de Participación Ciudadana del Estado de Yucatán (Ipepac), 2012, “Acta de sesión extraordinaria con carácter de urgente del 6 de julio de 2012", 6 de julio, en línea: <http://www.ipepac.org.mx/actas/2012/SESION-EXTRAORDINARIA-CON-CARACTER-DE-URGENTE-DEL-06-DEJULIO-DE-2012.pdf>.

Instituto Federal Electoral (IFE), 2008, Análisis comparativo de la reforma electoral constitucional y legal 20072008, Instituto Federal Electoral, México.

2009, El sistema de cómputo de votos en elecciones federales, documento informativo, Instituto Federal Electoral, México.

_ 2012a, "CG244/2012. Acuerdo del Consejo General del Instituto Federal Electoral por el que se emiten los lineamientos para la sesión especial de cómputo distrital del Proceso Electoral Federal 2011-2012", 25 de abril, Instituto Federal Electoral, en línea: <http:// www.ife.org.mx/docs/IFE-v2/DS/DS-CG/DS-SesionesCG/CG-acuerdos/2012/Abril/CGor201204-25/ CGo250412ap15.pdf>.

2012b, "CG301/2012. Acuerdo del Consejo General del Instituto Federal Electoral por el que se aprueba el programa de fiscalización a los partidos políticos y coaliciones propuesto por la Unidad de Fiscalización, respecto de los ingresos y gastos de campaña, relativos a los candidatos a la presidencia de los Estados Unidos Mexicanos para el Proceso Electoral Federal 2011-2012 y se aprueba la presentación anticipada del Dictamen Consolidado y Proyecto de Resolución de la misma elección presidencial", 16 de mayo, Instituto Federal Electoral, en línea: <http://www.ife.org. $\mathrm{mx} /$ docs/IFE-v2/DS/DS-CG/DS-SesionesCG/CGacuerdos/2012/Mayo/CGext201205-16/CGe160512ap9.pdf $>$.

, 2012c, "CG383/2012. Acuerdo del Consejo General del Instituto Federal Electoral por el que se aprueba el cuadernillo de consulta para votos válidos y votos nulos para el desarrollo de la sesión especial de cómputos distritales", 7 de junio, Instituto Federal Electoral, en línea: <http://www.ife.org.mx/docs/IFE-v2/DS/DSCG/DS-SesionesCG/CG-acuerdos/2012/junio/ CGext201206-07_01/CGe70612ap6.pdf>.

_ 2012d, "CG487/2012. Acuerdo del Consejo General del Instituto Federal Electoral por el cual se da respuesta al escrito de la Coalición denominada 'Movimiento Progresista', 3 de julio, Instituto Federal Electoral, en línea: <http://www.ife.org.mx/docs/IFE-v2/ DS/DS-CG/DS-SesionesCG/CG-acuerdos/2012/Julio/CGext201207-03/CGe030712apunico.pdf>.

, 2013a, "CG31/2013. Resolución del Consejo General del Instituto Federal Electoral respecto del procedimiento de queja en materia de fiscalización de los recursos de los partidos políticos nacionales, instaurado en contra de los Partidos Revolucionario Institucional y Verde Ecologista de México, integrantes de la otrora Coalición 'Compromiso por México', identificado como Q-UFRPP 58/12 y sus acumulados Q-UFRPP 246/12 y Q-UFRPP 232/12", 23 de enero, Instituto Federal Electoral, en línea: <http://www.ife.org.mx/docs/ IFE-v2/CNCS/CNCS-IFE-Responde/2013/01\%20 Enero/rmonex/P74eng.pdf $>$.

, 2013b, "CG49/2013. Acuerdo del Consejo General del Instituto Federal Electoral, por el que se ordena la devolución del 'Dictamen Consolidado de la Unidad de Fiscalización de los Recursos de los Partidos Políticos y proyecto de resolución del Consejo General del Instituto Federal Electoral respecto de las irregularidades encontradas en el dictamen consolidado de la revisión anticipada de los informes de ingresos y gastos de campaña, relativos a los candidatos a la presidencia de los Estados Unidos Mexicanos para el Proceso Electoral Federal 2011-2012', a la Unidad de Fiscalización de los Recursos de los Partidos Políticos", 6 de febrero, Instituto Federal Electoral, en línea: <http://www.ife.org.mx/docs/IFEv2/DS/DS-CG/DS-SesionesCG/CG-acuerdos/2013/Febrero/CGext201302-06/CGe60213ap3.pdf>, p. 6. 
, 2013c, "Versión estenográfica de la sesión extraordinaria del Consejo General del Instituto Federal Electoral, celebrada en el salón de sesiones del propio Instituto", 30 de enero, Instituto Federal Electoral, en línea:<http://www.ife.org.mx/docs/IFE-v2/CNCS/ CNCS-VersionesEstenograficas/2013/01\%20Enero/ VESE300113.pdf>.

—, 2013 d, "Versión estenográfica de la sesión extraordinaria del Consejo General del Instituto Federal Electoral, realizada en la Sala de Consejo del propio Instituto", 6 de febrero, Instituto Federal Electoral, en línea: <http://www.ife.org.mx/docs/IFE-v2/CNCS/CNCSVersionesEstenograficas/2013/01\%20Enero/VESE300113.pdf>.

Muñoz Oliveira, Luis, 2011, La razonabilidad, virtud de la democracia, Porrúa, México.

Olvera, Alberto J., 2008, Ciudadanía y democracia, Instituto Federal Electoral (Cuadernos de Divulgación de la Cultura Democrática, núm. 27), México.

Pérez Vázquez, Carlos, 2010, “Ensayo general”, en Jorge Alcocer y Lorenzo Córdova, Democracia y reglas del juego, 2010, Instituto de Investigaciones Jurídicas, Universidad Nacional Autónoma de México, México.

Secretaría de Gobernación (Segob), 2008, "Decreto por el que se expide el Código Federal de Instituciones y Procedimientos Electorales", en Diario Oficial de la Federación, 14 de enero, Poder Ejecutivo de la Federación, México.

Taniguchi, Hanako, 2012, "El Tribunal Electoral confirma una diputación más a favor del PRI”, en CNN México, 15 de agosto, en línea: <http://mexico.cnn.com/nacional/ 2012/08/15/el-tribunal-electoral-confirma-una-diputacion-mas-a-favor-del-pri>.

Tribunal de Justicia Electoral y Administrativa del Estado de Yucatán (Teeyuc), 2012, "Recurso de inconformidad, exp. RI-062/2012", 11 de agosto, en línea: <http:// www.teeyuc.org.mx/acceso/resoluciones/2012/RI/RI062-2012.pdf>.

Tribunal Federal del Poder Judicial de la Federación (Trife), 2012a, "Juicio de inconformidad, exp. ST-JIN-19/2012", 31 de julio, en línea: <http://www.te.gob.mx/salasreg/ ejecutoria/sentencias/toluca/ST-JIN-0019-2012.pdf>.

, 2012b, "Recurso de reconsideración, exp. SUPREC-126/2012”, 15 de agosto, en línea: <http://portal. te.gob.mx/colecciones/sentencias/html/SUP/2012/ REC/SUP-REC-00126-2012.htm>.

, 2012c, "Juicio de inconformidad, exp. SM-JIN38/2012", 21 de julio, en línea: <http://www.te.gob.mx/ salasreg/ejecutoria/sentencias/monterrey/SM-JIN0038-2012-Inc1.pdf>.

, 2012d, "Cómputo final, calificación jurisdiccional de la elección, declaración de validez y de presidente electo de los Estados Unidos Mexicanos", en Diario Oficial de la Federación, 6 de septiembre, Poder Ejecutivo de la Federación, México, en línea: <http:// dof.gob.mx/nota_detalle.php?codigo $=5267061 \& \mathrm{fec}$ ha $=06 / 09 / 2012>$.

, 2012e, "Recurso de reconsideración, exp. SUPREC-93/2012”, 23 de julio, en línea: <http://portal.te. gob.mx/colecciones/sentencias/html/SUP/2012/REC/ SUP-REC-00093-2012.htm>. 